\title{
Enabling Measurements of Qubit Errors at NEXUS
}

\author{
Alexander Novara, University of Pittsburgh; Sami Lewis, Daniel Bowring, Fermi National Laboratory
}

\section{Motivation}

As qubit technology progresses, one intriguing application involves their use as low noise single photon detectors. By measuring how the readout frequency of a resonator coupled to the qubit shifts in the presence of a photon, faint signals can be distinguished from mK-scale thermal noise. With this sensitivity, phenomena such as axion conversion to photons may be detectable.

$a$

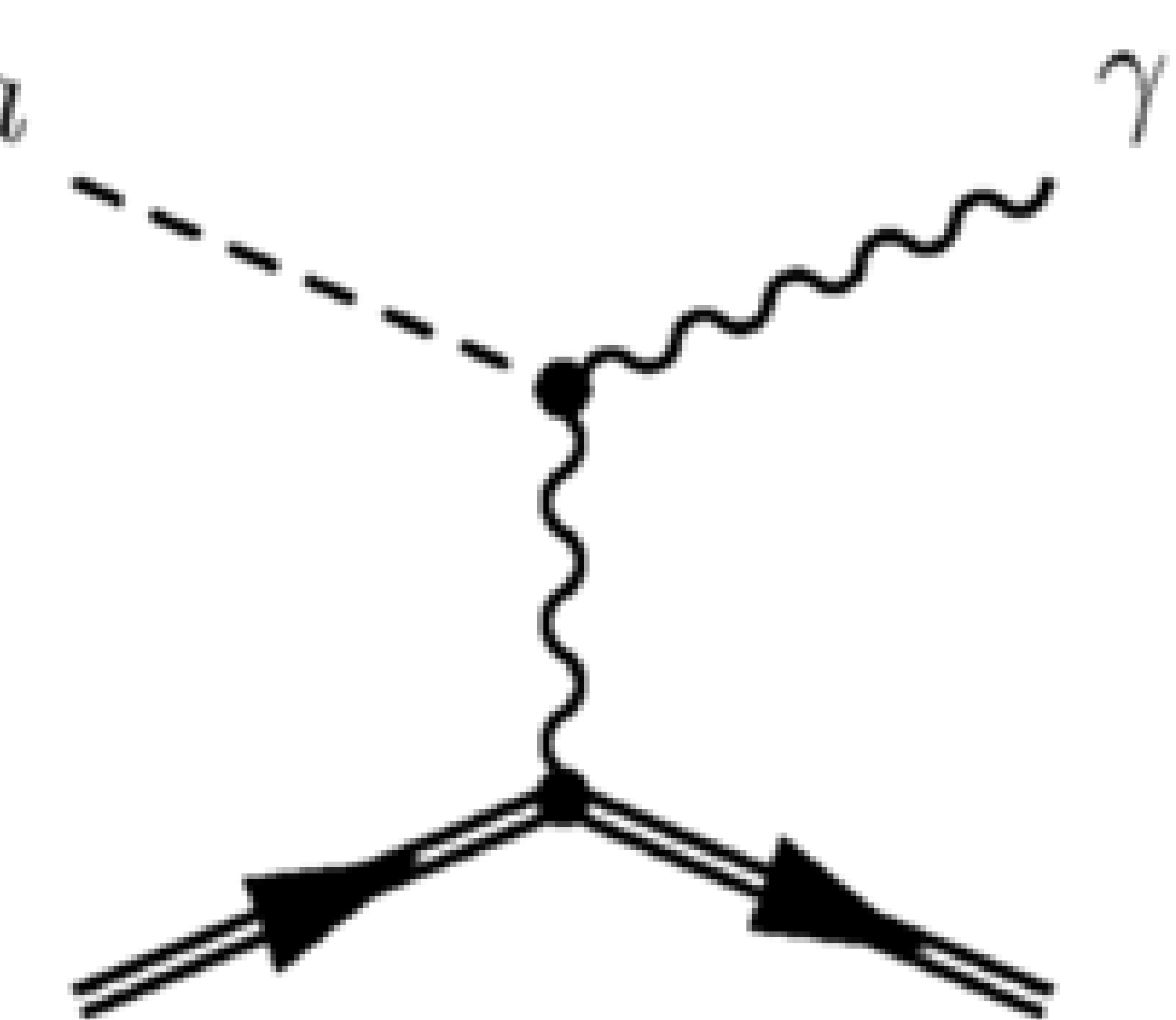

$\gamma$ Axion conversion to photon: ongoing and future experiments will explore regions of axion parameter space that correspond to Though they have long been viewed a promising dark matter candidate, some DMplausible axion mass values have thus far
been difficult to probe via conventionat photon counting techniques due to weak signal strength relative to noise.

\section{Objective}

Recent scholarship suggests that ionizing radiation may cause major issues in qubits, namely decoherence and 'catastrophic' correlated errors across time and space. Compromised coherence constrains all qubit applications while such multi-qubit errors break current error-correction algorithms. We are using NEXUS-Northwestern EXperimental Underground Site at Fermilab-to measure the impact of a low-radiation environment on qubit experiments. If a material difference in coherence time and/or error rate is detected, future qubit applications may need to be designed with such conclusions in mind.
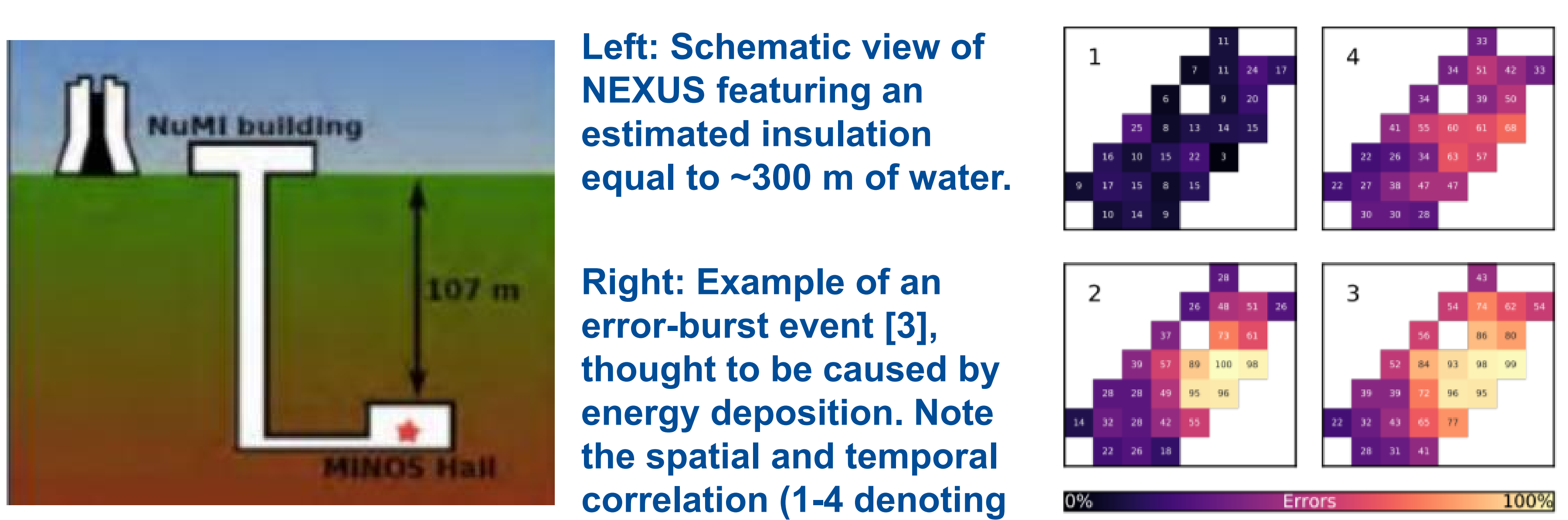

the spatial and temporal
correlation $(1-4$ denoting time slices).

\section{Qubit Spectroscopy}

To investigate these errors, we prepare and monitor quantum states. By transmitting tailored signals across the feedline shared by our 4 qubit-resonator pairs, we can identify errors, track their correlations, and monitor their impact on coherence time. The figure below is taken from a collaborator's paper [1] and depicts a set of pulses they employed; we soon will repeat the procedure using precisely the same chip. This controlled study should ensure that any differences in error rate and associated deleterious effects will represent evidence to quantify the impact of radiation shielding on qubit operations.

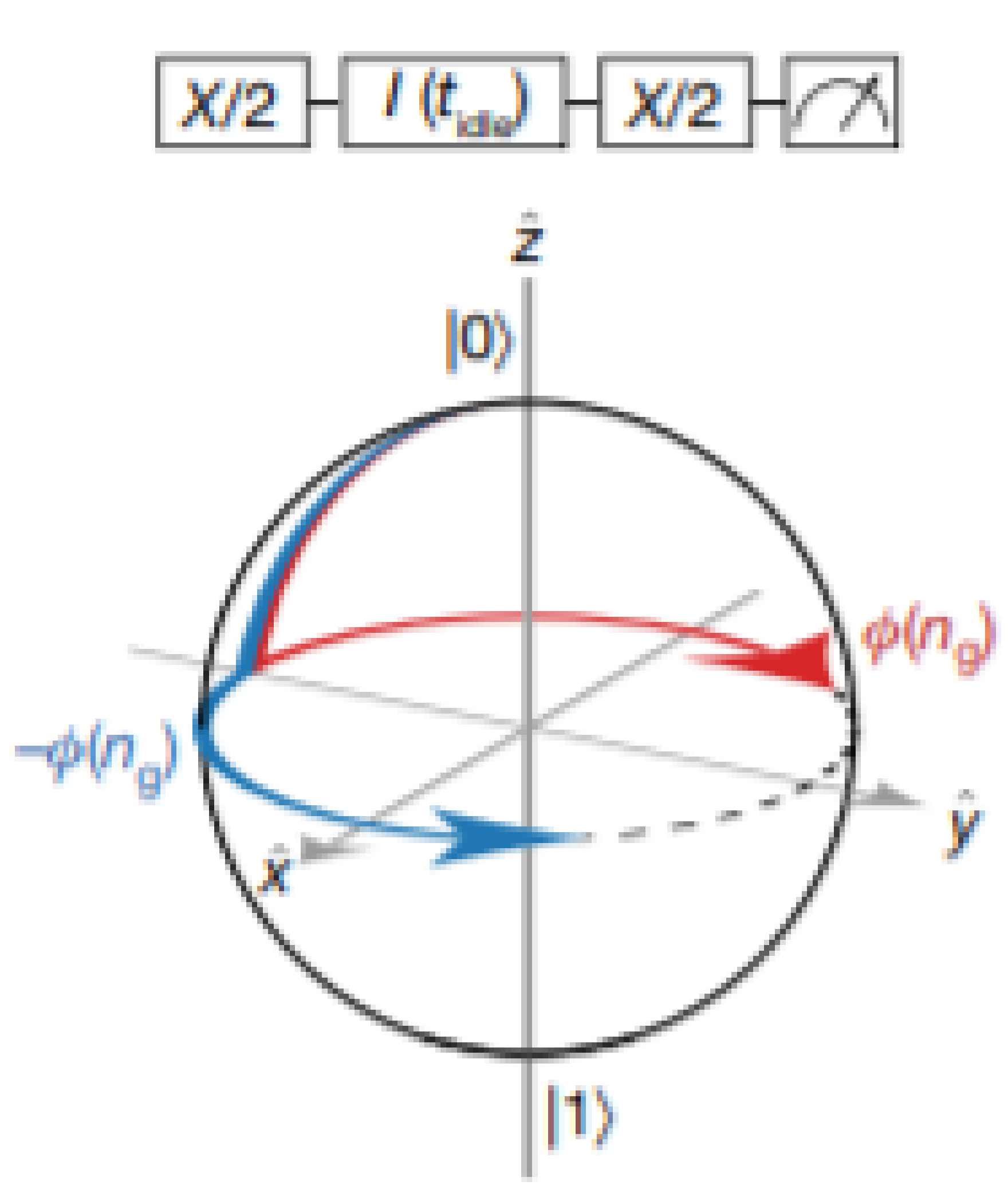

his panel [1] depicts a pulse sequence we will "listener' for charge bursts indicative of deposition by ionizing radiation. It is prepared in he $|0\rangle$ state and induced to transition to the $|1\rangle$ tate via a tuned pulse-idle-pulse sequence. The 政 the offset charge on the qubit, which remains Wughly constant under ordinary operation. When a burst occurs, however, the pulse res due to the new charge level. Abovancy (with the same qubits) a strong correlation was observed between large charge jumps in nearby qubits, and the rate of bursts was noted. If that correlation persists yet error events are less
frequent, it is likely that ionizing radiation is noticeably harming qubit function. Additionally. measurements of coherence time during bursts will be conducted with the same equipment.

\section{Acknowledgements}

This manuscript has been authored by Fermi Research Alliance, LLC under Contract No. DE-AC02-07CH11359 with the U.S. Department of Energy, Office of Science, Office of High Energy Physics.

\section{References}

[1] Wilen, C.D. et al. Correlated charge noise and relaxation errors in superconducting qubits. Nature 594, 369-373 (2021). https://doi.org/10.1038/s41586-021-03557-5 [2] Krantz, P. et al. A Quantum Engineer's Guide to Superconducting Qubits. Applied ows 6, 021318 (2019). http://dx.doi.org/10.1063/1.5089550

[3] McEwen, M. et al. Resolving catastrophic error bursts from cosmic rays in large

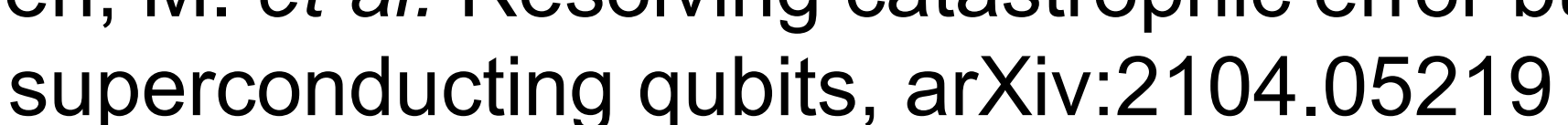

[4] Bratrud, G., Bowring, D. Qubit Signal Processing and the Search for Dark Matter, FERMILAB-POSTER-21-109-STUDENT

\section{Measurement Methods}

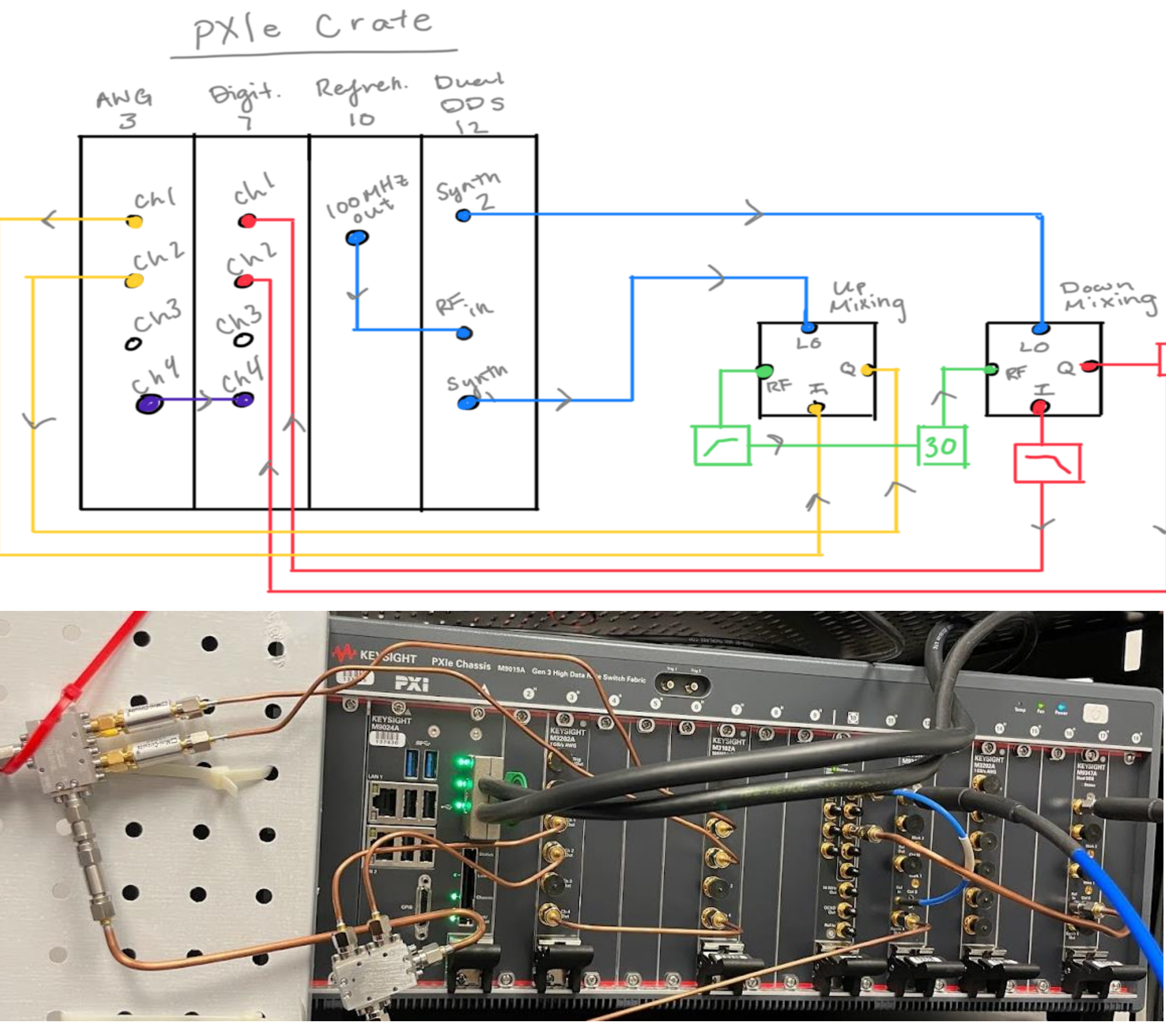

The setup [4] used qubit control electronics. Signal flows from the waveform generator to an I/Q mixer
which translates the pulse frequency into the qubit's

resonator's readout frequency using the
Keysight DDS Keysight DDS
synthesizer as a local oscillator.
From there, any From there, any
response is downmixed and digitized to be stored and analyzed

But how do we send these pulses? We developed control protocols for the 'warm electronics' shown above. Each instrument was tested and configured to-as an ensemble-generate arbitrary pulses such as gaussianenvelope wave packets that are used to prepare desired states or readout pulses to measure them. The pictured configuration allowed us to test input and output procedures and verify that everything is working as intended before adding the fridge and qubits into the loop. The $30 \mathrm{~dB}$ attenuator between the mixers acts as a stand-in for the qubit payload in this test setup.

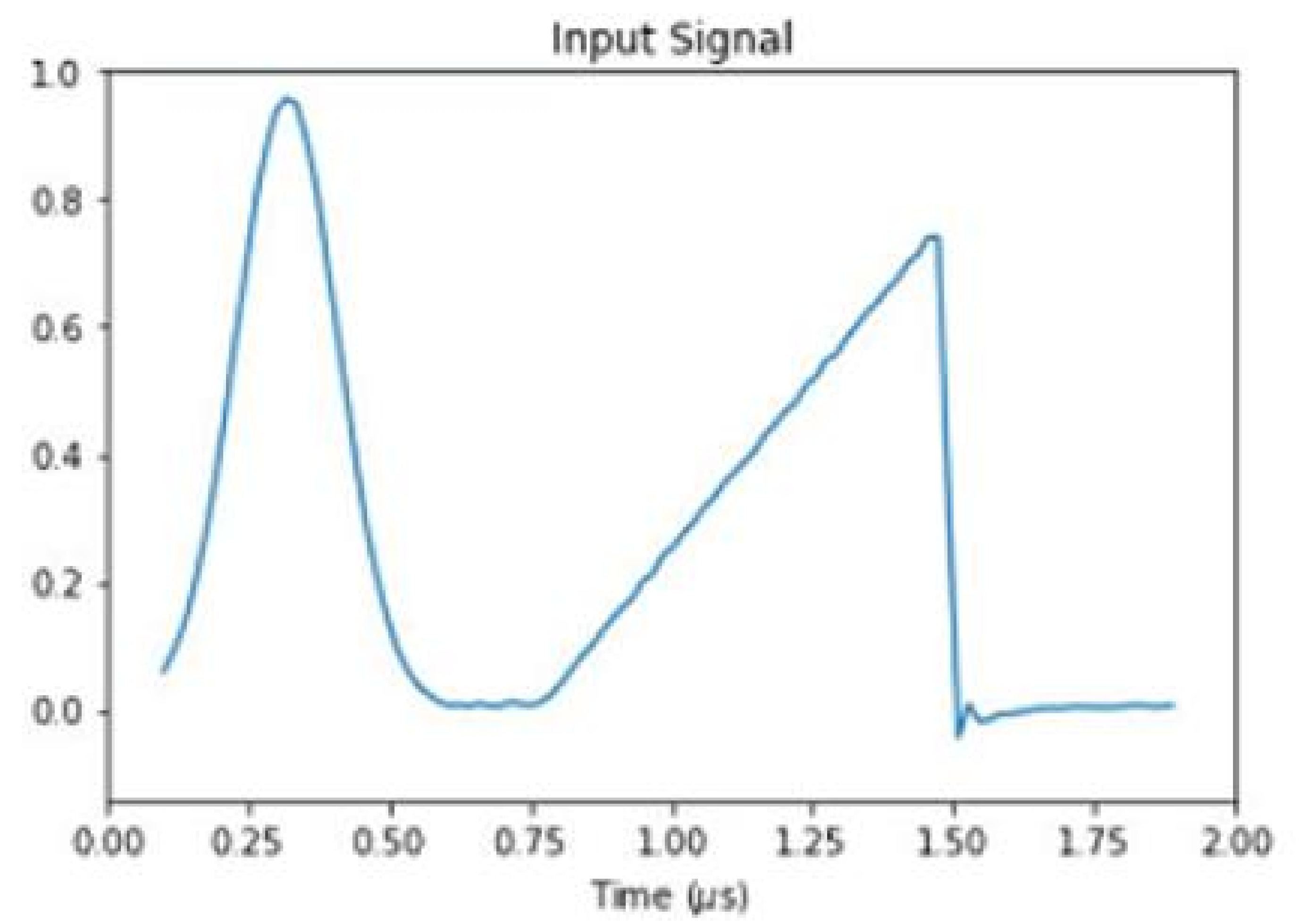

plot of a custom waveform sent directly Digitizer simulating an input and output cycle. With this verification successful, we are pulsed qubit spectroscopy. All the electronic
quipment is controllable programmatically: sitrary pulses can be and/or measure the qubit energy levels and their excess charges. 